\title{
0438 BICYCLE RIDING IN ISRAEL
}

K Peleg, M Siman-Tov* Correspondence: Gertner Institute for Epidemiology \& Health Policy Research. National Center for Trauma \& Emergency Medicine Research, Tel Hashomer 52621, Israel

10.1136/ip.2010.029215.438

Background Bicycle riding has become a popular form of recreation and sport. Safety and injury prevention related to bicycling in Israel is unknown.

Objectives To characterise cycling behaviours among adults in Israel.

Methods An observational study was performed and questionnaires were completed for adult cyclists, ages 18 years and older. Data were collected in May 2008 and focused on riding characteristics, bicycle-related injuries and use of protective gear. Comparisons were performed based on cycling type offroad, road, leisure (park) and transportation-related.

Results Observations were conducted on 3945 cyclists and questionnaires were completed for 679 cyclists. Off-road and road cyclists had more years of cycling than leisure cyclists,

tended to ride in groups rather than alone (95\% vs 75\%, respectively), rode more often during the week ( $40 \%$ vs $24 \%$, respectively) and for longer periods of time. Differences in the use of safety accessories were also observed. Self-reported injuries differed by cycling type. Half of the road cyclists reported injuries over the past 3 years, which was 1.5 times greater than for off-road cyclists and 2.5 times greater than for leisure cyclists. Conclusions Bicycle riders are considered a high-risk group vulnerable to injury. This study should be used as a basis to promote safe-cycling as well as for developing appropriate intervention programs in an effort to reduce bicycle-related injuries. 